\title{
Ursodeoxycholic Acid Modifies Gut-Derived Endotoxemia in Neonatal Rats
}

\author{
SARAH JANE SCHWARZENBERG AND MARY BUNDY \\ Department of Pediatrics. University of Minnesota. Minneapolis, Minnesota 5545.5
}

\begin{abstract}
We developed a model for the translocation of intraluminal endotoxin in the neonatal animal and used it to examine the capacity of a nonhepatotoxic bile acid, ursodeoxycholic acid (UDCA), to modify endotoxin translocation and cytokine response. Three-d-old SpragueDawley rats were randomized to receive enterally either no drug, lipopolysaccharide (LPS, $1 \mathrm{mg} /$ animal), or UDCA $(400 \mu \mathrm{g} /$ animal) alone, or UDCA followed by LPS $1 \mathrm{~h}$ later. One $h$ after LPS administration, the rats were killed and plasma endotoxin and tumor necrosis factor (TNF) were measured. Control animals had low circulating endotoxin (21.2 \pm 7.6 endotoxin units) and TNF $(0.06 \pm 0.02$ $\mathrm{ng} / \mathrm{mL}$ ). Enteral administration of LPS $1 \mathrm{~h}$ before the rats were killed resulted in significant elevation of endotoxin $(249.5 \pm 71.3, p=0.008)$ and TNF $(3.6 \pm 1.3, p=0.019)$. UDCA alone did not alter endotoxin levels $(8.7 \pm 2.1)$. UDCA $1 \mathrm{~h}$ before LPS prevented the rise in endotoxin (38.9 \pm 11.2 endotoxin units) and TNF $(0.2 \pm 0.05)$ significantly. Chenodeoxycholic acid was studied in a similar group of experiments and prevented neither the translocation of LPS nor the development of increased TNF levels in animals receiving LPS. In conclusion, LPS can cross the intestinal barrier in the normal neonatal rat. UDCA, administered before LPS, can decrease the translocation of LPS and prevent the cytokine response as measured by TNF levels. We speculate that UDCA, administered prophylactically, might reduce morbidity in clinical conditions leading to gut-derived endotoxemia. ( $\mathrm{Pe}$ diatr Res 35: 214-217, 1994)
\end{abstract}

\section{Abbreviations}

EU, endotoxin unit

LPS, lipopolysaccharide

TNF, tumor necrosis factor

UDCA, ursodeoxycholic acid

Endotoxin, a component of the outer membrane of gramnegative bacteria, is well established as a major mediator of vascular injury, leading to multisystem organ failure and death (1). The systemic effects of circulating endotoxin are mediated predominantly through the stimulation of mononuclear cells to produce TNF, IL-1, IL-6, colony-stimulating factors, and eicosanoids. Of these mediators, TNF has been shown to play a central role in the development of systemic hypotension and vascular insufficiency, which initiates many of endotoxin's adverse effects (2).

The biologically active portion of endotoxin is LPS, a complex

Received June 16, 1993; accepted September 28. 1993.

Correspondence: Dr. Sarah Jane Schwarzenberg. Box 274 UMHC, Minneapolis, MN 55455.

Supported by a grant from Ciba-Geigy Corp.. Summit, NJ, which also provided the UDCA used in the experiment. structure of $\mathrm{O}$-specific polysaccharides, core polysaccharides, and lipid A. Parenterally administered purified LPS reproduces the systemic effects of injected endotoxin (2). For the purposes of this study, we will use the term LPS to refer to purified bacterial lipopolysaccharides and the term endotoxin to refer to the crude outer membrane of gram-negative bacteria. This is a modification of the convention of van Deventer et al. (3).

The large reservoir of gram-negative bacteria in the intestine has been implicated as a source of endotoxin in several clinical conditions in adults (4). Similar studies in newborns and premature infants have suggested that gut-derived endotoxemia may play a role in the complications of sepsis, necrotizing enterocolitis, and total parenteral nutrition-associated cholestasis in this age group (5-7). In this study, we establish a model to study gutderived endotoxemia in neonatal animals and use the model to examine a therapy to prevent translocation of endotoxin. Intraluminal bile acids have been suggested as a treatment for endotoxemia in adults (8). UDCA is a relatively hydrophilic bile acid with fewer potential side effects than traditional ox bile preparations (9). Our study shows that UDCA can reduce or prevent gut-derived endotoxemia in neonatal animals. This represents a first step toward assessing the potential for this drug to modify the outcome of human neonatal disease associated with increased endotoxin translocation.

\section{MATERIALS AND METHODS}

Materials. All chemicals were purchased from Sigma, St. Louis, MO, unless otherwise noted. LPS was derived from Escherichia coli serotype 055:B5, phenol extracted.

Animals. All animal studies were approved by the University of Minnesota Animal Care and Use Committee. Sprague-Dawley rat dams were purchased from Taconic, Germantown, NY. Within $24 \mathrm{~h}$ of delivery, litters were equalized by cross-fostering and culling to produce litters of approximately 10 pups each. At $3 \mathrm{~d}$, pups were separated from their mothers for $1 \mathrm{~h}$ and kept warm and dry. After $1 \mathrm{~h}$ of starvation, LPS, UDCA (provided by Ciba-Giegy Corp., Summit, NJ), chenodeoxycholic acid, or a combination of these was administered according to the research protocol via an orogastric tube of $1.78 \mathrm{~mm}$ external diameter (Tygon tubing). LPS was administered in a dose of $1 \mathrm{mg} / \mathrm{animal}$ ( $100 \lambda$ in normal saline with $1.0 \%$ bromphenol blue), UDCA at $400 \mu \mathrm{g} /$ animal ( $10 \lambda$ in ethanol), and chenodeoxycholic acid at $400 \mu \mathrm{g} /$ animal ( $10 \lambda$ in ethanol). The orogastric tube was removed between treatments. Animals were killed by decapitation, and plasma was collected and frozen at $-20^{\circ} \mathrm{C}$.

Endotoxin and TNF assays. LPS was measured in plasma using a chromogenic limulus amoebocyte lysate test (BioWhittaker Inc., Walkersville, MD). The detection limit is 0.1 EU. All samples were measured in at least two dilutions; all dilutions were run in duplicate. We demonstrated that concentrations of UDCA 4-fold those of LPS did not affect the results of the assay (OD for $25 \mathrm{pg}$ LPS in $\mathrm{NaCl}$ was 1.106 ; with $0.1 \mathrm{ng}$ of UDCA in ethanol, it was $1.16 \pm 0.021)$. TNF was measured by ELISA (Genzyme Corp., Cambridge, MA). All data were 
analyzed by one-way analysis of variance and Fischer protected least significant difference on a Macintosh computer with Statview (Abacus Concepts. Inc., Berkeley. CA). Results are expressed as the mean \pm SEM.

\section{RESULTS}

Animal weights and response to orogastric feedings. The mean animal weight at $3 \mathrm{~d}$ was $9.3 \pm 0.1 \mathrm{~g}(n=98)$ (no weight was recorded for two animals). Between experimental groups, there was no significant difference in pup weights, except for that of the group receiving LPS alone $(9.0 \pm 0.3 \mathrm{~g})$ compared with the group receiving UDCA alone $(10.4 \pm 0.6 \mathrm{~g})$. However, linear regression analysis detected no correlation between pup weight and circulating endotoxin levels $\left(R^{2}=0.18\right)$, indicating that the difference in weight had no influence on endotoxin levels. Correct placement of the orogastric tubes was assessed by the appearance of a blue blush in the animals' stomachs during infusion. Animals tolerated orogastric feedings well, although they exhibited temporary lethargy after the insertion of the orogastric tube.

Enteral LPS is translocated into the bloodstream in infant rats. Figure 1 shows the levels of circulating endotoxin in neonatal rats administered enteral LPS. Rats who received no LPS had negligible levels of LPS in their plasma (21.2 $\pm 7.6 \mathrm{EU}, n=12)$. Rats who received LPS $1 \mathrm{~h}$ before they were killed had significantly elevated levels of plasma endotoxin $(249.5 \pm 71.3 \mathrm{EU}, n$ $=17, p=0.008$ ). Although plasma endotoxin levels were elevated in animals receiving LPS 2 and $4 \mathrm{~h}$ before the rats were killed. they were not significantly different from those of controls.

UDCA decreases LPS translocation from intestine in infant rats. To determine whether enteral UDCA could suppress translocation of enteral endotoxin into the bloodstream, we examined animals receiving UDCA 1 or $2 \mathrm{~h}$ before administration of LPS. Figure 2 shows that animals receiving UDCA alone had very low levels of circulating endotoxin $(8.7 \pm 2.1 \mathrm{EU}, n=6)$. These levels were no different than those of animals receiving no endotoxin. Animals receiving UDCA $1 \mathrm{~h}$ before administration of LPS $(2 \mathrm{~h}$ before they were killed) had levels of circulating endotoxin (38.9 $\pm 11.2, n=14$ ) no different from animals receiving UDCA alone

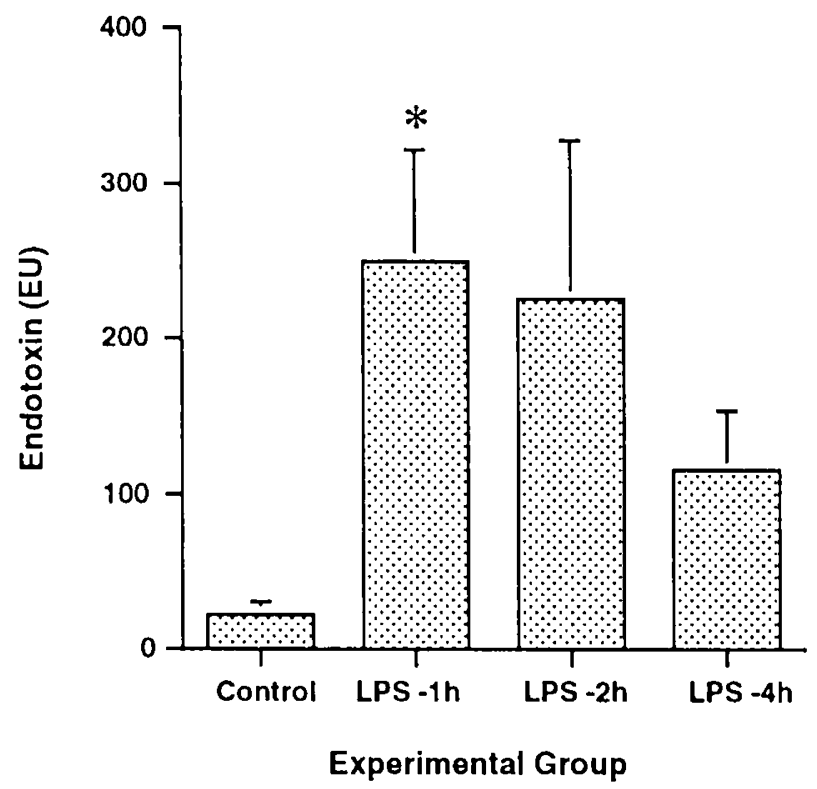

Fig. 1. Endotoxin levels in neonatal animals after enteral LPS. Experimental group 1 (control) received no therapy $(n=12)$, group 2 received LPS $1 \mathrm{~h}$ before they were killed ( $n=17$ ), group 3 received LPS $2 \mathrm{~h}$ before they were killed $(n=4)$, and group 4 received LPS $4 \mathrm{~h}$ before they were killed $(n=4)$. Significant differences from the control group (group 1) are marked with an asterisk.

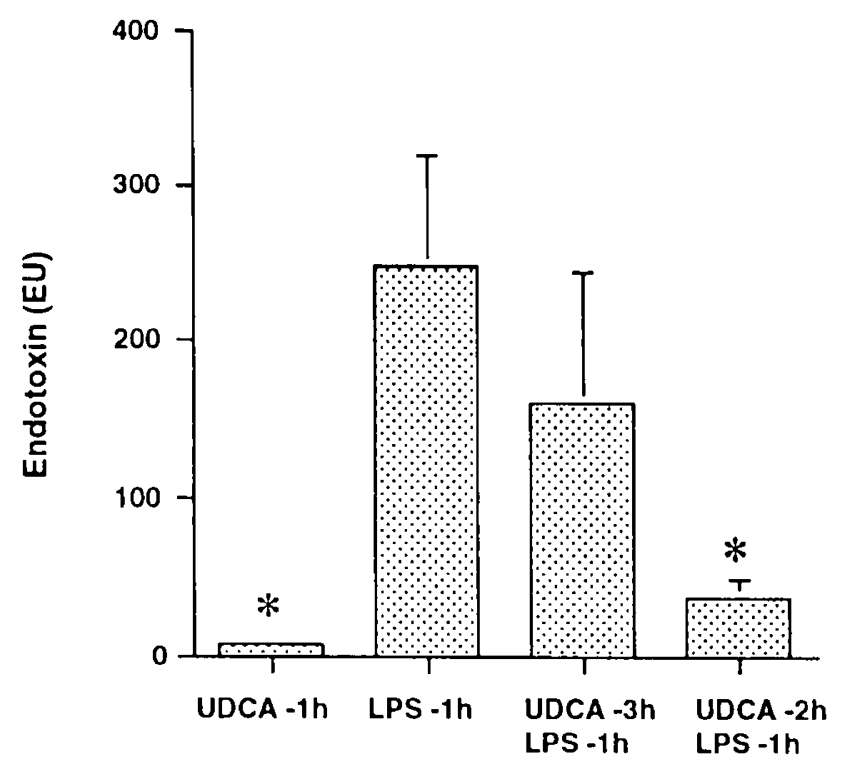

Experimental Group

Fig. 2. Endotoxin levels in neonatal animals treated with UDCA before LPS. Experimental group 1 received UDCA alone, $1 \mathrm{~h}$ before they were killed, $n=6$ : group 2 received LPS alone 1 h before they were killed ( $n=17$ ) (these are the same animals as in Fig. 1, group 2), group 3 received UDCA $2 \mathrm{~h}$ before LPS or $3 \mathrm{~h}$ before they were killed $(n=6)$. and group 4 received UDCA $1 \mathrm{~h}$ before LPS or $2 \mathrm{~h}$ before they were killed $(n=14)$. Significant differences from the LPS only control group are marked with an asterisk.

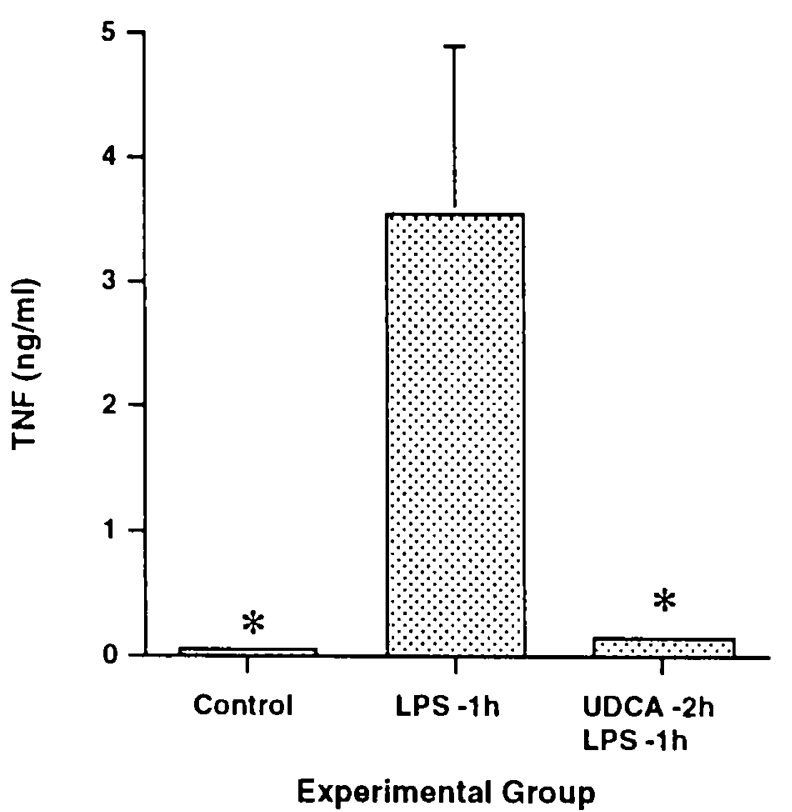

Fig. 3. Plasma TNF levels in neonatal animals after enteral LPS and UDCA. Experimental group 1 (control) received no treatment $(n=6)$. group 2 received LPS alone $(n=9)$, and group 3 received UDCA $1 \mathrm{~h}$ before LPS or $2 \mathrm{~h}$ before they were killed $(n=7)$. Significant differences from the LPS only control group are marked with an asterisk.

and significantly different from those of animals receiving LPS alone $(p=0.007)$. Administration of UDCA $2 \mathrm{~h}$ before LPS ( 3 $\mathrm{h}$ before they were killed) had no effect on circulating LPS levels.

$U D C$ A decreases $T N F$ production in rats receiving enteral $L P S$. Because TNF is the major mediator of the systemic effects of endotoxin, we examined whether enterally administered UDCA could inhibit the effects of circulating endotoxin to increase plasma TNF levels. As shown in Figure 3, control animals had 
negligible plasma levels of TNF $(0.06 \pm 0.02 \mathrm{ng} / \mathrm{mL}, n=6)$. Administration of enteral LPS $1 \mathrm{~h}$ before the rats were killed significantly increased circulating TNF levels $(3.6 \pm 1.3 \mathrm{ng} / \mathrm{mL}$, $n=9, p=0.019$ ). Administration of UDCA $2 \mathrm{~h}$ before administration of LPS reduced levels of TNF to levels similar to those of the control animals $(0.2 \pm 0.05 \mathrm{ng} / \mathrm{mL}, n=7)$.

Chenodeoxycholic acid fails to reduce LPS translocation. To determine whether a more hydrophobic bile acid, chenodeoxycholic acid, could produce results similar to those seen with UDCA, we examined the effect of chenodeoxycholic acid feeding on enteral translocation of LPS. Results are shown in Figure 4. Rats fed chenodeoxycholic acid alone had circulating levels of endotoxin similar to those of rats fed LPS alone $(69.7 \pm 20.7$ EU, $n=12$ compared with $146.4 \pm 43.8 \mathrm{EU}, n=9, p=0.15$ ). Administration of chenodeoxycholic acid before administration of LPS had no effect on circulating levels of endotoxin (156.4 \pm $52.9 \mathrm{EU}, n=8$ ). Similar results were obtained for plasma TNF levels (chenodeoxycholic acid alone, $0.7 \pm 0.1 \mathrm{ng} / \mathrm{mL}, n=6$; LPS alone $0.6 \pm 0.1 \mathrm{ng} / \mathrm{mL}, n=3$; and chenodeoxycholic acid before administration of LPS, $0.9 \pm 0.2 \mathrm{ng} / \mathrm{mL}, n=4 ; p=0.4$ ).

\section{DISCUSSION}

Gut-derived endotoxemia has been implicated in a spectrum of adverse effects ranging from isolated hepatic or renal injury to vascular insufficiency leading to multisystem organ failure and death $(1,4,8)$. The common factor linking diseases associated with gut-derived endotoxemia is increased transit of endotoxin from the gut lumen, seen in diseases resulting in intestinal degeneration or inflammation, ethanol ingestion, or intestinal vascular insufficiency (4). Gut-derived endotoxemia has also been seen in cholestatic conditions, including bile duct obstruction (8) or prolonged administration of total parenteral nutrition (10).

In neonates, endotoxemia has been linked to complications in several diseases. Scheifele $e t$ al. have shown that endotoxemia is present in neonates with gram-negative bacterial sepsis (11), small-bowel overgrowth (12), and necrotizing enterocolitis (13). This group specifically demonstrated that the thrombocytopenia associated with necrotizing enterocolitis was correlated with the

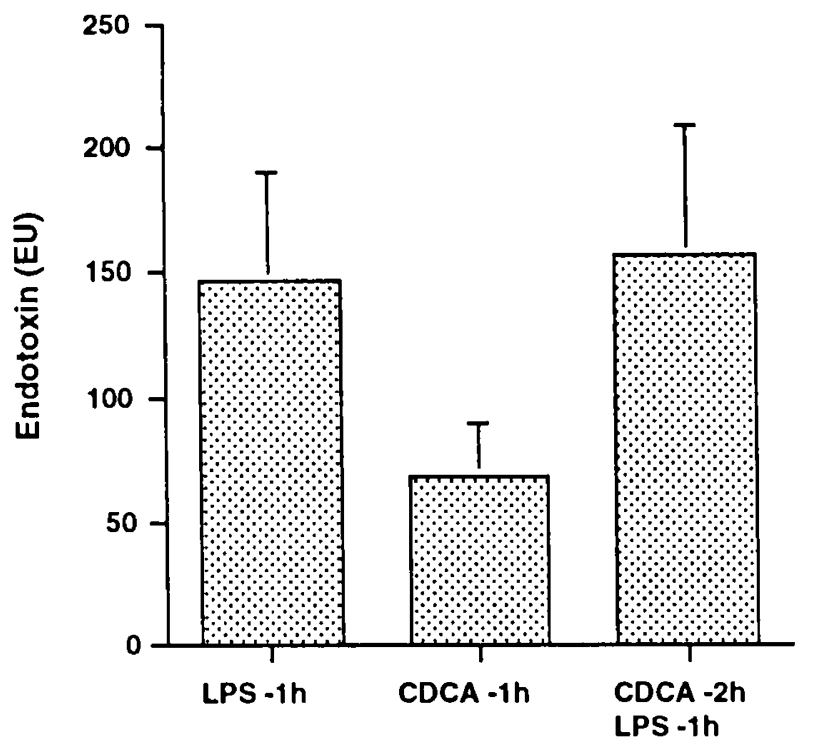

Experimental Group

Fig. 4. Endotoxin levels in neonatal animals treated with chenodeoxycholic acid $(C D C A)$ before LPS. Experimental group 1 received LPS alone $1 \mathrm{~h}$ before they were killed $(n=9)$, group 2 received CDCA alone $\mathrm{I} \mathrm{h}$ before they were killed $(n=12)$, and group 3 received CDCA $1 \mathrm{~h}$ before LPS or $2 \mathrm{~h}$ before they were killed $(n=8)$. No significant differences were seen among any of the three groups. presence of circulating endotoxin (14). Endotoxemia may also be a factor in the development of total parenteral nutritionassociated cholestasis in neonates (7). Experimental studies in rats have led to the suggestion that endotoxin might contribute to the development of giant cell or neonatal hepatitis (15). Although in some cases the source of endotoxemia in neonates is an abscess or another extragastrointestinal source, many times the source is gut-derived endotoxin (5).

For several reasons, neonates may experience both increased translocation of endotoxin from the intestine and increased complications associated with circulating endotoxin compared with adults. The neonatal small bowel can take up macromolecules more readily than the adult small bowel. This uptake is nonselective, probably the result of pinocytosis. Although many of these macromolecules are destroyed within the enterocyte, some reach the systemic circulation (16). If large quantities of endotoxin are present in the neonatal small bowel, there may be an increased risk of endotoxin translocating into the systemic circulation. Secondly, neonates have lower intraluminal bile salt concentrations than do adults, with premature infants particularly affected $(7,17,18)$. Because bile acids appear to disrupt endotoxin (19), reducing its translocation from the intestine $(8$. 20 ), factors that reduce intestinal bile salt concentration may increase the risk of endotoxin translocation. These physiologic factors may in part explain the observations of Scheifele $e t$ al. (6), who detected endotoxemia in $60 \%$ of neonates admitted to a tertiary care facility. Normal levels of luminal bile acids and low levels of macromolecule uptake by the gut in adult rats may explain why endotoxin is not passively absorbed from the gut in normal adult rats (20) and why it is necessary to induce malnutrition, burns, or hypotension to induce bacterial translocation in adult rats (21).

In addition, the clinical response to endotoxemia is different in neonates compared with adults. Zeller et al. (22) showed that 10-d-old rats were 300 times as sensitive to the effects of endotoxin, as measured by mortality, as 28 -d-old rats. Several developmental factors could account for this increased sensitivity to the effects of circulating endotoxin, including reduced reticuloendothelial system activity in the neonate (23) and differences in neonates compared with adults in the glucoregulatory and hemodynamic regulatory changes associated with endotoxemia (5).

Because of the differences between neonates and adults in their capacity to translocate endotoxin and their responses to systemic endotoxin, it is important to study the effects of gut-derived endotoxemia directly in the neonate. Our study showed that neonatal rats can serve as a model for the uninduced absorption of endotoxin in the newborn. This model provided an opportunity to examine the role of bile acids in preventing gut-derived endotoxemia in neonatal rats. In particular, we were interested in developing a therapy that might be safe and useful over the long periods of time that premature and full-term infants could be at risk for endotoxemia.

Several lines of evidence suggest that intraluminal bile acids might prevent translocation of endotoxin. Kocsar et al. (20) showed that sodium deoxycholate could decrease the absorption of endotoxin from the peritoneal cavity of rats by $47 \%$. Sodium deoxycholate also prevented the absorption of enterally administered tritiated endotoxin in rats. This was thought to be caused by the capacity of sodium deoxycholate to fragment endotoxin into smaller, nontoxic particles (19), although it is not clear why these smaller fragments would not be absorbed. Studies are needed to determine the mechanism by which bile acids modify intestinal absorption of endotoxin, whether the effect is the result of direct action of the bile acid on the endotoxin or the result of alterations in intestinal motility or absorptive capacity. Because endotoxin was implicated in renal failure associated with the postoperative management of patients with obstructive jaundice, Cahill (24) administered sodium deoxycholate $48 \mathrm{~h}$ before surgery to a group of patients with obstructive jaundice. The treated group had less endotoxemia and reduced incidence of renal 
impairment compared with untreated patients. These results are similar to those of Evans et al. (25) using sodium taurocholate. Thompson et al. (26) found less portal vein endotoxemia, but no change in renal impairment, in patients with obstructive jaundice treated with UDCA compared with untreated controls (26).

Because UDCA, unlike more hydrophobic bile acids, has not been shown to be cytotoxic (9), we chose to study this bile acid as a prophylaxis against endotoxin translocation in neonatal animals. We demonstrated that UDCA was effective in eliminating gut-derived endotoxemia if given $1 \mathrm{~h}$ before administration of endotoxin. This is in agreement with the study by Gaeta et al. (27), who showed that UDCA could reduce portal and peripheral blood endotoxin levels in adult rats with biliary obstruction. In our study, UDCA did not reduce circulating endotoxin if administered $2 \mathrm{~h}$ before endotoxin administration. This may provide a clue to the mechanism by which UDCA acts, because it suggests that UDCA and endotoxin must be present simultaneously for UDCA to prevent passage of endotoxin into the systemic circulation. We also showed that reducing the translocation of endotoxin from the gut leads to the elimination of circulating TNF in these animals. Thus. UDCA can prevent the systemic effects of gut-derived endotoxemia in these animals.

Interestingly, we found that chenodeoxycholate, a more lipophilic bile acid, did not reduce either the transit of endotoxin into the systemic circulation or TNF levels. One possible explanation for this is that chenodeoxycholic acid causes intestinal irritation, or increased gut permeability, leading to increased levels of macromolecular translocation.

Our results suggest the possibility that the UDCA might be valuable in preventing total parenteral nutrition-associated hepatic injury or some complications associated with necrotizing enterocolitis or sepsis in infants. Several caveats to this conclusion should be noted. First, the dose of UDCA used in this study was large; it remains to be determined what threshold dose is capable of preventing endotoxin translocation. Second, UDCA is metabolized to lithocholate by intestinal bacteria (28) and neonates are deficient in the sulfation detoxification pathway for lithocholate (17). Despite the lower levels of intestinal anaerobic bacteria in the first month of life, there is a theoretical concern that UDCA might be hepatotoxic in neonates. Little study of this phenomenon has been done even in adults, and research in this area is hampered by several things: first, not all humans possess intestinal bacteria that produce lithocholate; second. much of the intestinal lithocholate is not absorbed from the intestine; and third, many individuals being treated with UDCA had abnormal serum bile acid profiles before UDCA was initiated because of underlying liver disease (28). Nonetheless, this potential problem must be considered. Finally, many neonates on long-term total parenteral nutrition have short-gut syndrome. UDCA may not be absorbed in patients with short-gut syndrome, although this should have no effect on its capacity to inhibit translocation of intestinal endotoxin. Of greater concern would be the potential induction of diarrhea by UDCA, seen in a small number of treated patients, which might reduce enteral nutrition absorption.

Although significant progress has been made in the antibiotic therapy of infectious diseases in neonates, several problems remain. One of the most challenging is the control of the inflammatory cascade that accompanies infection and that is not eliminated by killing the infectious agent (23). At least a part of this cascade is precipitated by gut-derived endotoxemia. Our studies raise the speculation that UDCA, ir administered prophylactically to neonates, might reduce mortality and morbidity in clinical conditions leading to endotoxin translocation.
Acknowledgments. The authors thank Dr. Randall Burd and Dr. David Dunn for instruction in performing the endotoxin assays and Dr. Harvey L. Sharp for valuable discussions.

\section{REFERENCES}

1. Kraft R, Ruchti C. Burkhardt A. Cottier H 1992 Pathogenic principles in the development of gut-derived infectious-toxic shock (GITS) and multiple organ failure. Curr Stud Hematol Blood Transfus 59:204-240

2. Doran JE 1992 Biological effects of endotoxin. Curr Stud Hematol Blood Transfus 59:66-99

3. van Deventer SJH, ten Cate JW. Tytgat GNJ 1988 Intestinal endotoxemia clinical significance. Gastroenterology 94:825-831

4. Nolan JP 1989 Intestinal endotoxins as mediators of hepatic injury: an idea whose time has come again. Hepatology 10:887-89!

5. Zeller WP, Goto M. Hurley RM 1988 Endotoxin in newborn septic shock: significance, metabolic, and cardiovascular changes. Ann Clin Lab Sci 18:253-259

6. Scheifele DW. Olsen E, Fussell S. Pendray M 1985 Spontaneous endotoxinemia in premature infants: correlations with oral feeding and bowel dysfunction J Pediatr Gastroenterol Nutr 4:67-74

7. Balistreri WF, Bove KE 1990 Hepatobiliary consequences of parenteral nutrition. Prog Liver Dis 9:567-601

8. Bailey ME 1976 Endotoxin, bile salts and renal function in obstructive jaundice. Br J Surg 63:774-778

9. Hofmann AF 1993 The enterohepatic circulation of bile acids in health and disease. In: Sleisenger MH. Fordtran JS (eds) Gastrointestinal Disease: Pathophysiology, Diagnosis, Management. WB Saunders. Philadelphia, pp $127-$ 149

10. Pappo I, Becovier H, Berry EM. Freund HR 1991 Polymyxin B reduces cecal flora. TNF production and hepatic steatosis during total parenteral nutrition in the rat. J Surg Res 51:106-112

11. Scheifele DW, Melton P. Whitchelo V 1981 Evaluation of the Limulus test for endotoxemia in neonates with suspected sepsis. J Pediatr 98:899-903

12. Scheifele DW, Melton PW. Ebelt V 1981 Endotoxinaemia per se as cause of neonatal morbidity. [letter] Lancet 1:337

13. Scheifele DW 1990 Role of bacterial toxins in neonatal necrotizing enterocolitis. J Pediatr 117(Pt 2):S44-S46

14. Scheifele DW. Olsen EM, Pendray MR 1985 Endotoxinemia and thrombocytopenia during neonatal necrotizing enterocolitis. Am J Clin Pathol 83:227229

15. Andres JM. Walker WA 1979 Effect of Escherichia coli endotoxin on the developing rat liver. I. Giant cell induction and disruption in protein metabolism. Pediatr Res 13:1290-1293

16. Walker WA. Isselbacher KJ 1974 Uptake and transport of macromolecules by the intestine: possible role in clinical disorders. Gastroenterology 67:531550

17. Suchy FJ. Bucuvalas JC. Novak DA 1987 Determinants of bile formation during development: ontogeny of hepatic bile acid metabolism and transport. Semin Liver Dis 7:77-84

18. Balistreri WF, Heubi JE, Suchy FJ 1983 Immaturity of the enterohepatic circulation in early life: factors predisposing to "physiologic" maldigestion and cholestasis. J Pediatr Gastroenterol Nutr 2:346-354

19. Rudbach JA, Anacker RL, Haskins WT, Johnson AG. Milner KC. Ribi E 1966 Physical aspects of reversible inactivation of endotoxin. Ann NY Acad Sci 133:629-643

20. Kocsar LT. Bertok L, Varteresz V 1969 Effect of bile acids on the intestinal absorption of endotoxin in rats. J Bacteriol 100:220-223

21. Deitch EA. Winterton J. Berg R 1987 Effect of starvation, malnutrition, and trauma on the gastrointestinal tract flora and bacterial translocation. Arch Surg 122:1019-1024

22. Zeller WP. Goto M. Witek-Janusek L. Hurley RM 1991 Mortality, tempora substrate and insulin responses to endotoxic shock in zero, ten and twentyeight day old rats. Surg Gyn Obst 173:375-383

23. Levin M 1990 The inflammatory response to infections. In: de Louvois J. Harvey D (eds) Infection in the Newborn. John Wiley \& Sons, West Sussex. England. pp $1-12$

24. Cahill CJ 1983 Prevention of postoperative renal failure in patients with obstructive jaundice: the role of bile salts. Br J Surg 70:59()-595

25. Evans HJR, Torre-Alba V. Hudd C. Knight M 1982 The effect of preoperative bile salt administration on postoperative renal function in patients with obstructive jaundice. Br J Surg 69:706-708

26. Thompson JN, Cohen J, Blenkharn JI. McConnell JS, Barr J. Blumgart LH 1986 A randomized clinical trial of oral ursodeoxycholic acid in obstructive jaundice. Br J Surg 73:6.34-636

27. Gaeta GB. Van Bossuyt H, Desmaretz C, Wisse E 1989 Mechanisms of endotoxemia in liver diseases: new experimental evidence. In: Wisse $\mathrm{E}$ Knook DL, Decker K (eds) Cells of the Hepatic Sinusoid. Vol. 2. Kupffer Cell Foundation. Rijswijk, the Netherlands, pp 343-344

28. Javitt NB 1992 Ursodeoxycholic acid therapy: the baby and the bathwater. [editorial] Hosp Pract (Off Ed) 27:12-16 